\title{
Quantum nondemolition of the Universe
}

\author{
Brian P. Dolan* \\ Department of Mathematical Physics, St. Patrick's College, Maynooth, Ireland \\ and School of Theoretical Physics, Dublin Institute for Advanced Studies, 10 Burlington Road, Dublin 4, Ireland
}

(Received 31 August 1992; revised manuscript received 7 May 1993)

\begin{abstract}
The role of decohering histories in a quantum description of the Universe is examined. A sufficient condition for different histories to decohere is derived, the cosmological nondemolition condition. This condition automatically ensures that quantum histories decohere regardless of the initial wave function of the Universe.

PACS number(s): 04.60. $+\mathrm{n}$, 03.65.Bz, 98.80.Hw
\end{abstract}

The problem of constructing and interpreting a consistent quantum description of the whole Universe has occupied many workers since the inception of the quantum theory. One school of thought holds that quantum mechanics in itself cannot be a complete theory of the world because the measurement process necessarily involves a classical apparatus which cannot be described purely in terms of quantum mechanics [1]. Yet others hold that, interpreted correctly, the quantum theory is complete in itself as a description of the physical world [2]. There has recently been an increase of interest in the cosmological aspects of this question as people are beginning to enquire into a quantum description of the genesis of the Universe (for a recent review see Halliwell [3]). Indeed this has led to a debate as to the form of the initial wave function of the Universe [4,5].

Nevertheless many physicists still feel uneasy about the interpretation of these ideas and, in an attempt to alleviate this uneasiness, a fresh view of quantum mechanics has recently been proposed by Gell-Mann and Hartle [6], based on the notion of decoherence (see also [7]). Their approach is an attempt to put Everett's many worlds interpretation of quantum mechanics [8] on a more solid foundation and is based on the work of Griffiths and Omnes $[9,10]$. The concept of decoherence has been of growing importance in the understanding of quantum mechanics in recent years; see, e.g., [11].

The purpose of this article is to exhibit a method of explicitly constructing a large set of such decohered histories for any given system. We shall give sufficient (but not necessary) conditions for a set of histories to decohere, which we shall call the cosmological nondemolition (CND) conditions.

A history in the approach of Gell-Mann and Hartle [6] consists of an initial state in a Hilbert space, $\left|\psi_{0}\right\rangle$, together with a time series of projection operators in the Heisenberg picture:

$$
P_{m}\left(t_{m}\right), P_{m-1}\left(t_{m-1}\right), \ldots, P_{1}\left(t_{1}\right)
$$

with $t_{m}>t_{m-1}>\cdots>t_{1}$. More generally the initial

\footnotetext{
*Electronic address: bdolan@maths.may.ie
}

state can be replaced by an initial density matrix $\rho_{0}$ representing a mixed state. In the sequel we shall always assume that the initial state is pure, $\rho_{0}=\left|\psi_{0}\right\rangle\left\langle\psi_{0}\right|$, but this is not necessary for any of our arguments which all go through without change for an initial state which is mixed.

A set of histories comprises an exhaustive and exclusive, but not necessarily complete, set of such projection operators at each time $t_{i}$ for every value of $1 \leq i \leq m$, $\left\{P_{i}^{\alpha_{i}}\left(t_{i}\right) ; \alpha_{i}=1, \ldots, N_{i}\right\}$. In equation form

$$
\sum_{\alpha_{i}=1}^{N_{i}} P_{i}^{\alpha_{i}}\left(t_{i}\right)=1 \text { and } P_{i}^{\alpha_{i}}\left(t_{i}\right) P_{i}^{a_{i}^{\prime}}\left(t_{i}\right)=\delta_{\alpha_{i}, \alpha_{i}^{\prime}} P_{i}^{\alpha_{i}}\left(t_{i}\right) \text {. }
$$

Here $1 \leq \alpha_{i} \leq N_{i}$ labels the set of projection operators for each $t_{i}$. These sets might be different for each $i$, and can even consist of different numbers $N_{i}$ of projectors for each $i$. We shall abbreviate the notation by defining a vector, with integer coefficients $1 \leq \alpha_{i} \leq N_{i}$, on an $m$ dimensional lattice $\boldsymbol{\alpha}=\left(\alpha_{1}, \ldots, \alpha_{m}\right)$. The total number of such vectors is $N=N_{m} N_{m-1} \cdots N_{1}$. There are then $N$ histories for each initial state indexed by the vectors $\alpha$, one for each history:

$P(\alpha)\left|\psi_{0}\right\rangle=P_{m}^{\alpha_{m}}\left(t_{m}\right) P_{m-1}^{\alpha_{m-1}}\left(t_{m-1}\right) \cdots P_{1}^{\alpha_{1}}\left(t_{1}\right)\left|\psi_{0}\right\rangle$.

We shall call $\boldsymbol{P}(\boldsymbol{\alpha})$ a history operator. It is not necessarily a projection operator itself.

It is germane to the Gell-Mann-Hartle construction that their projection operators can project onto spaces of dimension greater than one; thus, it is possible that $N_{i}<\operatorname{dim} \mathscr{H}$ where $\operatorname{dim} \mathscr{H}$ is the dimension of the Hilbert

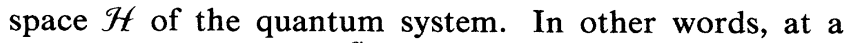
given time $t_{i}$ the set $\left\{P_{i}^{\alpha_{i}}\left(t_{i}\right)\right\}$ is not necessarily complete. Indeed, given such a set, Gell-Mann and Hartle define a coarse graining $\left\{P_{i}^{\beta_{i}}\left(t_{i}\right)\right\}$ where $\boldsymbol{\beta}$ belongs to a smaller lattice than $\alpha$ does with $1 \leq \beta_{i} \leq N_{i}^{\prime}, N_{i}^{\prime} \leq N_{i}$, and each $P_{i}^{\beta_{i}}\left(t_{i}\right)$ is a sum of some (possibly only one, but in general more than one) of the $P_{i}^{\alpha_{i}}\left(t_{i}\right)$ which is also exclusive, i.e., $P_{i}^{\beta_{i}}\left(t_{i}\right) P_{i}^{\beta_{i}^{\prime}}\left(t_{i}\right)=\delta_{\beta_{i}, \beta_{i}^{\prime}} P_{i}^{\beta_{i}}\left(t_{i}\right)$.

The observation behind the Gell-Mann-Hartle proposal is that our Universe appears classical to us; therefore, whatever its quantum history, it must be such that it is a 
close approximation to a classical history. They then seek a criterion for a subset of all possible quantum histories that will single them out as possible candidates for a universe that is a good approximation to a classical one.
The criterion that they adopt is a slightly more general version of one introduced by Griffiths [9]. They demand that the off-diagonal terms of the decoherence functional should vanish for every pair of histories in the set, i.e.,

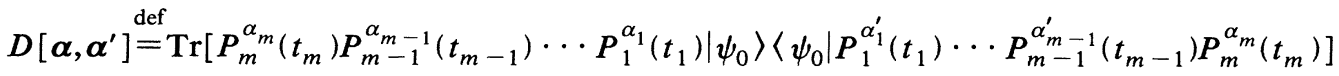

$$
\begin{aligned}
& =\left\langle\psi_{0}\left|P_{1}^{\alpha_{1}^{\prime}}\left(t_{1}\right) \cdots P_{m-1}^{\alpha_{m-1}^{\prime}}\left(t_{m-1}\right) P_{m}^{\alpha_{m}}\left(t_{m}\right) P_{m-1}^{\alpha_{m-1}}\left(t_{m-1}\right) \cdots P_{1}^{\alpha_{1}}\left(t_{1}\right)\right| \psi_{0}\right\rangle \\
& =\left\langle\psi_{0}\left|\boldsymbol{P}\left(\widetilde{\boldsymbol{\alpha}}^{\prime}\right) \boldsymbol{P}(\boldsymbol{\alpha})\right| \psi_{0}\right\rangle \\
& =0 \text {. }
\end{aligned}
$$

When this criterion holds, $P(\alpha)$ and $P\left(\widetilde{\alpha}^{\prime}\right)$ are said to decohere. [Griffiths adopted the slightly less stringent condition that only the real part of (3) hold [9].] We have adopted the notation $\widetilde{\boldsymbol{\alpha}}=\left(\alpha_{m}, \alpha_{m-1}, \ldots, \alpha_{1}\right)$, i.e., the vector $\alpha$ with the components in the reverse order. Clearly it is necessary for the final projection operators $P_{m}^{\alpha_{m}}\left(t_{m}\right)$ and $P_{m}^{\alpha_{m}^{\prime}}\left(t_{m}\right)$ to be equal (i.e., $\left.\alpha_{m}=\alpha_{m}^{\prime}\right)$ in order that this trace does not vanish trivially.

In practice the criterion of decoherence, (3), can be replaced by that of approximate decoherence. $P(\alpha)$ and $\boldsymbol{P}\left(\widetilde{\boldsymbol{\alpha}}^{\prime}\right)$ are said to approximately decohere if

$$
\left\langle\psi_{0}\left|P\left(\widetilde{\boldsymbol{\alpha}}^{\prime}\right) \boldsymbol{P}(\boldsymbol{\alpha})\right| \psi_{0}\right\rangle \approx 0 .
$$

The criterion that Gell-Mann and Hartle adopt for a set of histories to exhibit approximately classical behavior is that all different histories approximately decohere. This means that the $N \times N$ matrix $D\left[\alpha, \alpha^{\prime}\right]$, where $\alpha$ labels the rows and $\alpha^{\prime}$ labels the columns, is (approximately) diagonal. Given any history which has coherences, it is possible to coarse grain until the coherences disappear. The most refined coarse graining that eliminates coherences (there may be more than one) is a candidate for a set of classical histories which still retains a maximal amount of information about the system. Further coarse graining preserves decoherence, but loses more information about the initial state, until all $N_{i}=1$ and $P_{i}^{1}\left(t_{i}\right)=1$ and all information is lost.

Below we shall state a condition, the CND condition, which automatically leads to decoherence of histories. We shall see that this condition is very restrictive and, if adopted, puts a very tight constraint on possible histories in quantum gravity.

A consequence of the CND conditions will be that, after the time $t_{1}$, the system is in an eigenstate of all subsequent projections. This means that all subsequent projections after, but not necessarily including, $t_{1}$ do not disturb the state $P_{1}^{\alpha_{1}}\left(t_{1}\right)\left|\psi_{0}\right\rangle$. Hence they behave in a manner analogous to quantum nondemolition (QND) experiments [12] for all times after $t_{1}$. If we are dealing with a system which consists of the entire Universe and its possible histories we have a situation which corre- sponds to quantum nondemolition of the Universe, whence the phrase cosmological nondemolition (CND), which follows directly from our conditions below.

A second criterion in the Gell-Mann-Hartle approach for a quantum history to be a candidate for a good description of our classical Universe is that of the emergence of classical behavior for macroscopic bodies, but it is perhaps worthwhile bearing in mind that there may be some advantages to retaining some of the quantum behavior of the system in attempting to describe the macroscopic structure of our Universe. For example, it has been suggested that quantum correlations over large distances could be important in the description of the large scale structure of our Universe [13]. The CND hypotheses as presented here is not compatible with a completely classical description of the Universe; variables at successive moments of time are not completely correlated according to classical laws, but this may prove to be an advantage in the long run. There is still much to be understood at the interface between the classical and quantum worlds.

We shall first give some examples to illustrate our point. For the first example consider a free nonrelativistic particle of mass $M$ moving on the one dimensional real line with the coordinate $-\infty<x<\infty$. Firstly we shall take $m=2$ and $N_{1}=N_{2}=2$. Thus there are only two mutually exclusive and exhaustive projection operators at each of the two times $t_{1}$ and $t_{2}$, and hence four possible histories labeled by

$$
\boldsymbol{\alpha}=\left(\alpha_{1}, \alpha_{2}\right)=\left\{\begin{array}{l}
(1,1), \\
(1,2), \\
(2,1), \\
(2,2) .
\end{array}\right.
$$

We can use the exhaustive property of the projection operators to write

$$
P_{i}^{2}\left(t_{i}\right)=1-P_{i}^{1}\left(t_{i}\right) \stackrel{\text { def }}{=} \bar{P}_{i}^{1}\left(t_{i}\right) \text { for } i=1,2 .
$$

We shall define projection operators $P^{x}(t)$ by

$$
\begin{aligned}
P^{x}(t) & =e^{i \hat{H} t}|x\rangle\langle x| e^{-i \hat{H} t} \\
& =\frac{M}{2 \pi t} \int_{-\infty}^{\infty} d y \int_{-\infty}^{\infty} d y^{\prime} \exp \left\{i \frac{M}{2 t}\left[\left(x-y^{\prime}\right)^{2}-(x-y)^{2}\right]\right\}|y\rangle\left\langle y^{\prime}\right|,
\end{aligned}
$$


where $|y\rangle$ are an orthonormal basis of position eigenstates, $\left\langle y \mid y^{\prime}\right\rangle=\delta\left(y-y^{\prime}\right), \hat{H}=\hat{p}^{2} / 2 M$ is the Hamiltonian operator and $|x\rangle$ is a fixed position eigenstate with eigenvalue $x$. We have set $\hbar=1$.

Now we choose $P_{i}^{1}\left(t_{i}\right)=P^{x_{i}}\left(t_{i}\right)$ for two positions $x_{1}$ and $x_{2}$. A little work shows that, for $t_{1} \neq t_{2}$,

$$
\begin{aligned}
P_{x_{1}}\left(t_{1}\right) P_{x_{2}}\left(t_{2}\right)= & {\left[\frac{M}{2 \pi}\right]^{3 / 2} \frac{1}{\sqrt{t_{2} t_{1}\left(t_{2}-t_{1}\right)}} \exp \left\{i \frac{M}{2} \frac{\left(x_{2}-x_{1}\right)^{2}}{\left(t_{2}-t_{1}\right)}-i \frac{\pi}{4}\right\} } \\
& \times \int_{-\infty}^{\infty} d y \int_{-\infty}^{\infty} d y^{\prime} \exp \left\{-i \frac{M}{2}\left[\frac{\left(x_{2}-y\right)^{2}}{t_{2}}-\frac{\left(x_{1}-y^{\prime}\right)^{2}}{t_{1}}\right]\right\}|y\rangle\left\langle y^{\prime}\right|
\end{aligned}
$$

and

$$
P^{x_{1}}\left(t_{1}\right) P^{x_{2}}\left(t_{2}\right) P^{x_{1}}\left(t_{1}\right)=\left[\frac{M}{2 \pi}\right]^{2} \frac{1}{t_{1}\left(t_{2}-t_{1}\right)} \int_{-\infty}^{\infty} d y \int_{-\infty}^{\infty} d y^{\prime} \exp \left\{-\frac{i}{2} \frac{M}{t_{1}}\left[\left(y-x_{1}\right)^{2}-\left(y^{\prime}-x_{1}\right)^{2}\right]\right\}|y\rangle\left\langle y^{\prime}\right| .
$$

Now suppose the initial state is $\left|x_{0}\right\rangle$. Then it turns out that

$$
\begin{aligned}
D[(1,1),(2,1)] & =\left\langle x_{0}\left|P^{x_{1}}\left(t_{1}\right) P^{x_{2}}\left(t_{2}\right) P^{x_{2}}\left(t_{2}\right) \bar{P}^{x_{1}}\left(t_{1}\right)\right| x_{0}\right\rangle \\
& =\left\langle x_{0}\left|P^{x_{1}}\left(t_{1}\right) P^{x_{2}}\left(t_{2}\right)\right| x_{0}\right\rangle-\left\langle x_{0}\left|P^{x_{1}}\left(t_{1}\right) P^{x_{2}}\left(t_{2}\right) P^{x_{1}}\left(t_{1}\right)\right| x_{0}\right\rangle
\end{aligned}
$$

is never zero for finite times. For large times $t_{2}>t_{1}$ it tends to zero like $\approx 1 / \sqrt{t_{2} t_{1}\left(t_{2}-t_{1}\right)}$. Thus these histories never decohere for any finite values of $t_{2}>t_{1}$.

Now consider the two projection operators $P^{p}=|p\rangle\langle p|$ and $\bar{P}^{p}=1-P^{p}$ for some momentum eigenstate $|p\rangle$ with an eigenvalue $p$. Since the Hamiltonian commutes with the momentum operator these two projection operators are independent of time. Take a different set of histories, again with $m=2, N_{1}=2$, and $N_{2}=2$, but now

$$
\begin{array}{ll}
P_{1}^{1}\left(t_{1}\right)=e^{i \hat{H} t_{1}} P^{p_{1}} e^{-i \hat{H} t_{1}}=P^{p_{1}}, & P_{1}^{2}\left(t_{1}\right)=\bar{P}^{p_{1}}, \\
P_{2}^{1}\left(t_{2}\right)=e^{i \hat{H} t_{2}} P^{p_{2}} e^{-i \hat{H} t_{2}}=P^{p_{2}}, & P_{2}^{2}\left(t_{2}\right)=\bar{P}^{p_{2}} .
\end{array}
$$

It is easy to show that all four $\left\{P^{p_{i}}, \bar{P}^{p_{i}}\right\}$ commute with each other and, since $P^{p_{1}} \bar{P}^{p_{1}}=0$, it is automatic that these histories decohere for any initial state, as

$$
\boldsymbol{P}^{p_{1}} \boldsymbol{P}^{p_{2}} \overline{\boldsymbol{P}}^{p_{1}}=\overline{\boldsymbol{P}}^{p_{1}} \boldsymbol{P}^{p_{2}} \boldsymbol{P}^{p_{1}}=\boldsymbol{P}^{p_{1}} \overline{\boldsymbol{P}}^{p_{2}} \overline{\boldsymbol{P}}^{p_{1}}=\overline{\boldsymbol{P}}^{p_{1}} \overline{\boldsymbol{P}}^{p_{2}} \boldsymbol{P}^{p_{1}}=0,
$$

and hence all off-diagonal elements of the matrix $D\left[\boldsymbol{\alpha}, \boldsymbol{\alpha}^{\prime}\right]$ vanish.

This example is very simple because the projectors $P^{p}$ are independent of time. A less trivial example is the following, where a time dependent set of projectors is constructed which nevertheless still ensures decoherence because they commute at the times $t_{i}$ but not at all other times.

Consider a spin- $\frac{1}{2}$ particle in a constant magnetic field $\mathbf{B}$ in the $z$ direction. The relevant term in the Hamiltonian for the time evolution of the spin is $\hat{H}=\mathbf{B} \cdot \boldsymbol{\sigma}=\left(\begin{array}{cc}B & 0 \\ 0 & { }_{B}\end{array}\right)$, where we have set $e \hbar / c=1$. Define the projection operators in the $x$ direction by

$$
P_{x}^{ \pm}=\frac{1}{2}\left(1 \pm \sigma_{x}\right)=\frac{1}{2}\left[\begin{array}{cc}
1 & \pm 1 \\
\pm 1 & 1
\end{array}\right]
$$

Clearly these form an exhaustive set, since $1=P_{x}^{+}+P_{x}^{-}$ Their time evolution is given by

$$
e^{i \hat{H} t} P_{x}^{ \pm} e^{-i \hat{H} t}=\frac{1}{2}\left(\begin{array}{cc}
1 & \pm e^{2 i B t} \\
\pm e^{-2 i B t} & 1
\end{array}\right)
$$

It is straightforward to show that

$$
\left[P_{x}^{+}(t), P_{x}^{+}\left(t^{\prime}\right)\right]=-\frac{i}{2} \sin \left[2 B\left(t-t^{\prime}\right)\right]\left[\begin{array}{cc}
1 & 0 \\
0 & -1
\end{array}\right]
$$

and it immediately follows [since $\left.P^{-}(t)=1-P^{+}(t)\right]$ that all four commutators $\left[P_{x}^{ \pm}(t), P_{x}^{ \pm}\left(t^{\prime}\right)\right]$ vanish if and only if $t-t^{\prime}=r \pi / 2 B$, where $r$ is an integer.

As a consequence any pair $P_{i}^{\alpha_{i}}\left(t_{i}\right)$ with $N_{i}=2$, $\forall i=1, \ldots, m$ and $P_{i}^{\alpha_{i}}\left(t_{i}\right)=P_{x}^{ \pm}\left(t_{i}\right)$ will automatically decohere when all the differences $2 B\left(t_{i}-t_{j}\right) / \pi=r_{i j}$ are integers for every pair $\{i, j\}$, regardless of the initial state. This is simply due to the the fact that, if any $\alpha_{i} \neq \alpha_{i}^{\prime}$ in the matrix $D\left[\boldsymbol{\alpha}, \boldsymbol{\alpha}^{\prime}\right]$ when $r_{i j} \in \mathbb{Z} \forall i, j$, then we can commute $P_{i}^{\alpha_{i}}\left(t_{i}\right)$ through until it hits $P_{i}^{\alpha_{i}^{\prime}}\left(t_{i}\right)$ and destroys it since the projection operators at any given time $t_{i}$ are exclusive.

The foregoing example serves as a prototype for a further interesting case, that of the harmonic oscillator with characteristic frequency $\omega$ for a particle of mass $M$. The creation and annihilation operators are

$$
\hat{a}^{\dagger}=\left[\frac{M \omega}{2}\right]^{1 / 2} \hat{x}+i \frac{1}{\sqrt{2 M \omega}} \hat{p}
$$

and

$$
\hat{a}=\left(\frac{M \omega}{2}\right)^{1 / 2} \hat{x}-i \frac{1}{\sqrt{2 M \omega}} \hat{p},
$$

with $\left[\widehat{a}, \hat{a}^{\dagger}\right]=1$, and the Hamiltonian is

$$
\hat{H}=\frac{1}{2 M} \hat{p}^{2}+\frac{M}{2} \omega^{2} \hat{x}^{2}=\omega\left[\hat{a}^{\dagger} \hat{a}+\frac{1}{2}\right] .
$$


The Heisenberg picture leads to the time evolution

$$
\widehat{a}(t)=e^{-i \omega t} \hat{a}, \quad \hat{a}^{\dagger}(t)=e^{i \omega t} \widehat{a}^{\dagger} .
$$

We shall construct the Fock space of eigenstates from the ground state $|0\rangle$ by $|n\rangle=(1 / \sqrt{n})\left(a^{\dagger}\right)^{n}|0\rangle$. Note that our normalization is chosen so that $\langle n \mid n\rangle=1$. Now define the projection operators

$$
\begin{aligned}
P_{n}^{ \pm} & =\frac{1}{2}(|n+1\rangle \pm|n\rangle)(\langle n+1 \pm|\langle n|) \\
& =\frac{1}{2}\left[\begin{array}{cc}
1 & \pm 1 \\
\pm 1 & 1
\end{array}\right],
\end{aligned}
$$

where the two dimensional subspace spanned by $|n+1\rangle$ and $|n\rangle$ has been represented in matrix form. The $P_{n}^{ \pm}$ have time evolution

$$
P_{n}^{ \pm}(t)=\frac{1}{2}\left[\begin{array}{cc}
1 & \pm e^{i \omega t} \\
\pm e^{-i \omega t} & 1
\end{array}\right]
$$

Since $P_{n}^{+}(t)=1-P_{n}^{-}(t)$ we have an exhaustive set of two exclusive projections at time $t$ for each value of $n$. The analogy with $P_{x}^{ \pm}(t)$ for the spin- $\frac{1}{2}$ particle is obvious.

One finds again

$$
\left[P_{n}^{+}(t), P_{n}^{+}\left(t^{\prime}\right)\right]=-\frac{1}{2} \sin \left[\omega\left(t-t^{\prime}\right)\right]\left[\begin{array}{cc}
1 & 0 \\
0 & -1
\end{array}\right) \text {. }
$$

Thus if we have a set of $\left\{P_{n}^{ \pm}\left(t_{i}\right)\right\}$ at different times $t_{m}>t_{m-1}>\cdots>t_{1}$ then they are all mutually commuting provided $\omega\left(t_{i}-t_{j}^{\prime}\right) / \pi=r_{i j}$ are all integers $\forall i, j$. By the same argument as before, we see that any set of histories constructed in this way automatically decoheres provided all the $\omega\left(t_{i}-t_{j}\right) / \pi$ are integers.

One can show that

$$
\left[P_{n}^{ \pm}(t), P_{n^{\prime}}^{ \pm}\left(t^{\prime}\right)\right]=0, \quad \forall t, t^{\prime},
$$

provided $n^{\prime} \neq n \pm 1$ or $n$. Thus we can even let the occupation number $n$ depend on the times $t_{i}$ giving a set $\left\{n_{i}\right\}$, provided no two of the $n_{i}$ differ by unity, and the construction still gives decohered histories automatically independent of the initial state.

This model can be extended to a set of $\mathcal{N}$ independent oscillators, labeled by an integer $s=1, \ldots, \mathcal{N}$, with frequencies $\omega_{s}$. We will assume that every $\omega_{s}$ is a rational multiple of some basic frequency $\omega$. This assumption allows us to write each $\omega_{s}$ as $\omega_{s}=\left(p_{s} / q_{s}\right) \omega$ for two relatively prime integers $p_{s}$ and $q_{s}$. Let each oscillator have projection operators $P_{n_{i}^{s}}^{ \pm}\left(t_{i}\right)$, defined as above, at each $t_{i} \cdot n_{i}^{s}$ is an integer labeling a Fock state $\left|n_{i}\right\rangle$ for each oscillator $s$ at time $t_{i}$. There are $2 \mathcal{N}$ such projection operators at each $t_{i}, \mathcal{N}$ of the $P_{n_{i}^{s}}^{+}\left(t_{i}\right)$ plus their $\mathcal{N}$ complements $P_{n_{i}^{s}}^{-}\left(t_{i}\right)$. They can thus be represented by $(2 \mathcal{N} \times 2 \mathcal{N})$ dimensional matrices. The condition that these matrices all commute is that no pair of integers in the set $\left\{n_{i}^{s}\right\}$ differ by unity and that every $\omega_{s}\left(t_{i}-t_{j}\right) / \pi$ be an integer for all pairs $\left\{t_{i}, t_{j}\right\}$. This will be true if the difference of every pair $t_{i}-t_{j}$ is $\pi$ times a multiple of the largest integer from the set of denominators, i.e., $\max \left\{q_{1}, \ldots, q_{\mathcal{N}}\right\}$. Again any such system automatically gives rise to decoherent histories independently of any assumptions about the initial state.

Having presented these examples we now go on to elaborate on the concept of mutually commuting projection operators and the part they play in the GellMann-Hartle construction.

The basic idea is the following. Suppose a history consists of an initial state, $\left|\psi_{0}\right\rangle$, and a set of $m$ exhaustive and exclusive projection operators implemented at times $\left\{t_{1}, \ldots, t_{m}\right\}$, so that $N_{i}=N / m, \forall i$. Then, if the projection operators are mutually commuting at the times at which they are implemented (but not necessarily at all other times), they can be simultaneously diagonalized. In an eigenbasis of the projectors it is then clear that any product of projectors at different times $t_{i}, 1 \leq i \leq m$, must be diagonal with eigenvalues one or zero. (This does not have to be true at an arbitrary time, but only at times from the set $\left\{t_{i}\right\}$, also the eigenvalues of the projectors can change with time, e.g., a given projector might have one particular eigenvalue being 0 at $t_{i}$ and 1 at $t_{i+1}$.) Thus the history operator $P(\alpha)$, the product of projectors over the entire history, is now itself a projection operator onto a linear subspace of the whole Hilbert space of the quantum system. Different histories will result in different subspaces and if all of these subspaces are linearly independent of each other then the histories automatically decohere, since $P(\alpha) P\left(\alpha^{\prime}\right)=0$ for two different sets $\boldsymbol{\alpha} \neq \boldsymbol{\alpha}^{\prime}$. That these subspaces are linearly independent for different histories is automatic since the projections at each time $t_{i}$ are assumed to be exclusive.

Note that the decoherence of histories via this mechanism is independent of the initial state $\left|\psi_{0}\right\rangle$, only the probability of a given history will depend on $\left|\psi_{0}\right\rangle$. Thus, in this construction, the debate mentioned above between the Hawking and Hartle [4] and Vilenkin [5] proposals for the initial quantum state of the Universe loses some of its immediacy, though it would still be important to find the class of initial states which leads to a probability close to unity for the observed Universe (i.e., homogeneous Robertson-Walker universe).

Thus we postulate the following hypothesis.

Cosmological nondemolition hypothesis. The possible quantum histories of the Universe for any initial state $\left|\psi_{0}\right\rangle$ correspond to sets of mutually exclusive and exhaustive projection operators $\left\{P_{i}^{\alpha_{i}}\left(t_{i}\right)\right\}$ for each $i$, labeled by the $N=N_{1} N_{2} \cdots N_{m}$ distinct vectors $\alpha$, which are mutually commuting for all the specified times $t_{i}, 1 \leq i \leq m$.

The condition that the projection operators be mutually commuting at all the chosen times $t_{i}$ can be a very strong one. It can easily preclude the possibility of $P_{i}^{\alpha_{i}}\left(t_{i}\right)$ being complete, and thus force us into losing information about the initial state. It will certainly place a very strong restriction on the possible histories of the Universe and could conceivably even single out a unique history, leading to a unique prediction for the possible time evolution of our Universe.

In general coarse graining can only improve the commutation properties of the projection operators. For example consider a consistent history for a spin- $\frac{1}{2}$ particle 
with $m=2, N_{1}=3, N_{2}=2$, and

$$
\begin{aligned}
& P_{1}^{1}\left(t_{1}\right)=P_{x}, \quad P_{1}^{2}\left(t_{1}\right)=P_{z}, \quad P_{1}^{3}\left(t_{1}\right)=1-P_{x}-P_{z}, \\
& P_{2}^{1}\left(t_{2}\right)=P_{x}, \quad P_{2}^{2}\left(t_{2}\right)=1-P_{x},
\end{aligned}
$$

where $P_{x}=\frac{1}{2}\left(\begin{array}{ll}1 & 1 \\ 1 & 1\end{array}\right)$ and $P_{z}=\left(\begin{array}{ll}1 & 0 \\ 0 & 0\end{array}\right)$. This history is consistent but does not satisfy the CND condition, since neither $P_{1}^{2}\left(t_{1}\right)$ nor $P_{1}^{3}\left(t_{1}\right)$ commutes with $P_{2}^{1}\left(t_{2}\right)$ or $P_{2}^{2}\left(t_{2}\right)$. We now coarse grain to a new set of histories with $N_{1}^{\prime}=2, N_{2}^{\prime}=2$, and

$$
\begin{array}{ll}
P_{1}^{1^{\prime}}\left(t_{1}\right)=P_{1}^{1}\left(t_{1}\right), & P_{1}^{2^{\prime}}\left(t_{1}\right)=P_{1}^{2}\left(t_{1}\right)+P_{1}^{3}\left(t_{1}\right), \\
P_{2}^{1^{\prime}}\left(t_{2}\right)=P_{2}^{1}\left(t_{2}\right), & P_{2}^{2^{\prime}}\left(t_{2}\right)=P_{2}^{2}\left(t_{2}\right) .
\end{array}
$$

Now all projection operators in this new set commute with each other and the CND condition is satisfied.

It is possible to relax the constraints somewhat and demand that the projection operators be merely approximately commuting at the times $t_{i}$ (the approximate CND condition). By this we mean that the modulus of their off-diagonal components is much less than unity. After all, in their original proposal, Gell-Mann and Hartle only required approximate decoherence for a quantum history to be regarded as approximately classical. If the offdiagonal components are small then, in the Schrödinger picture, the Universe does not have much time to wander away from its state at $t_{i}$ to a different eigenstate of $P_{i+1}^{\alpha_{i+1}}\left(t_{i+1}\right)$ and thus will almost inevitably be projected to the same eigenstate at $t_{i+1}$. Indeed in perturbation theory, for small time intervals $\Delta t_{i}=t_{i+1}-t_{i}$ [that is small relative to the rate of change of $\left.P_{i}^{\alpha_{i}}\left(t_{i}\right)\right]$, rather than the probability of a transition being proportional to $\Delta t_{i}$ (Fermi's golden rule), it is proportional to $\left(\Delta t_{i}\right)^{2}$. Thus the rate of change is proportional to $\Delta t_{i}$ and vanishes for small $\Delta t_{i}$. This is the famous "watched pot" or quantum Zeno effect. Thus the approximate CND condition leads to approximate decoherence. A more detailed version of the ideas presented here will apear in Ref. [14].
[1] J. S. Bell, Phys. World 3, 33 (1990).

[2] K. Gottfried, Phys. World 4, 34 (1991).

[3] J. J. Halliwell, in Quantum Cosmology and Baby Universes, Proceedings of the Jerusalem Winter School, Jerusalem, Israel, 1990, edited by S. Coleman, J. Hartle, T. Piran, and S. Weinberg (World Scientific, Singapore, 1991).

[4] S. W. Hawking, in Astrophysical Cosmology, Proceedings of the Study Week on Cosmology and Fundamental Physics, edited by H. A. Bruck, G. V. Coyne, and M. S. Longair (Pontificia Academiae Scientarium, Vatican City, 1982); J. B. Hartle and S. W. Hawking, Phys. Rev. D 28, 2960 (1983); S. W. Hawking, Nucl. Phys. B239, 257 (1984).

[5] A. Vilenkin, Phys. Lett. 117B, 25 (1982); Phys. Rev. D 27 , 2848 (1983); 30, 509 (1984); 32, 2511 (1985); Nucl. Phys. B252, 141 (1985); Phys. Rev. D 33, 3560 (1986); 37, 888 (1988).

[6] M. Gell-Mann and J. B. Hartle, in Complexity, Entropy and The Physics of Information, edited by W. Zurek, Santa Fe Institute Studies in the Science of Complexity No. 8 (Addison-Wesley, Redwood City, CA, 1991); J. B. Hartle, in 12th International Conference on General Relativity and Gravitation, Proceedings, Boulder, Colorado, edited by B.
R. Iyer, A. Kembhavi, J. Narliker, and C. W. Vishveshwara (Cambridge University Press, Cambridge, England, 1990).

[7] H. K. Dowker and J. J. Halliwell, Phys. Rev. D 46, 1580 (1992).

[8] H. Everett III, Rev. Mod. Phys. 29, 454 (1957).

[9] R. Griffiths, J. Stat. Phys. 36, 219 (1984).

[10] R. Omnes, Rev. Mod. Phys. 64, 339 (1992).

[11] W. Zurek, Phys. Rev. D 24, 1516 (1981); 26, 1682 (1982); A. Caldeira and A. Leggett, Physica 121A, 587 (1983); E. Joos and H. D. Zeh, Z. Phys. 59, 223 (1985).

[12] V. B. Braginsky and Yu. I. Vorontsov, Usp. Fiz. Nauk. 114, 41 (1974) [Sov. Phys. Usp. 17, 644 (1975)]; V. B. Braginsky, Yu. I. Vorontsov, and K. S. Thorne, Science 209, 547 (1980).

[13] S. K. Blau and A. H. Guth, in 300 Years of Gravitation, edited by S. W. Hawking and W. Israel (Cambridge University Press, Cambridge, England, 1987).

[14] B. P. Dolan, in Proceedings of the 1st Meeting of the Buddhist Enquiry After Natural Order, Dundee, Scotland, 1993, edited by D. T. Menace (Thomson, Dundee, in press). 